\title{
Systemic Lupus Erythematosus presenting with Bullous lesion, Lupus Nephritis and Seizure
}

\author{
Mahesh Raj Sigdel*
}

Department of Internal Medicine, TU Teaching Hospital, Kathmandu, Nepal

\section{Accepted on \\ December $16^{\text {th }}, 2012$ \\ DOI Name \\ 10.3126/jaim.v2i1.7633 \\ Keywords \\ Bullous lesion, BSLE, systemic lupus erythematosus, nephritis, seizure \\ Citation \\ Sigdel MR. Systemic Lupus Erythematosus presenting with Bullous lesion, Lupus Nephritis and Seizure: A review. Journal of Advances in Internal Medicine 2013;02(01):17-20.}

\begin{abstract}
Development of bullous lesion is a rare cutaneous manifestation of systemic lupus erythematosus (SLE). Bullous eruptions in SLE are divided into three categories. Firstly, the lesions of SLE may blister. Secondly, a number of primarily blistering diseases have been associated with SLE.Thirdly, bullous SLE (BSLE), characterized bysub-epidermal blisters, IgG deposition at the dermoepidermal junction and autoantibodies against collagen VII, forms a distinct group. We report an18 years girl who met American College of Rheumatology diagnostic criteria for SLE presenting with bullous lesion and nephritis and developed seizure during the same admission. She had anti-nuclear antibody positive, anti-DsDNA antibody negative and serum complement, C3, level decreased. She was managed with steroid, hydroxychloroquine and cyclophosphamide to which her renal function improved significantly and the skin lesions remitted partially. We would also like to highlight the difficulties in establishing differential diagnoses of bullous lesion in SLE.
\end{abstract}

\section{INTRODUCTION}

Systemic lupus erythematosus (SLE) is a chronic autoimmune multisystem disease. ${ }^{1}$ Cutaneous lupus erythematosus (CLE) is present in over $75 \%$ of patients at some point during the course of SLE and is the second most frequent presenting symptom after joint involvement. ${ }^{2,3}$ According to Gilliam and Sontheimer the cutaneous lesions of lupus could be lupus specific and lupus nonspecific. ${ }^{1}$ Development of bullous lesion is a rare cutaneous manifestation of SLE. ${ }^{1}$ Bullous eruptions in SLE are divided into three categories namely. ${ }^{4}$

1. Blisters secondary to vacuolar degeneration of the basal layer of the epidermis in SLE.

2. SLE associated with primary blistering diseases like dermatitis herpetiformis, bullous pemphigoid, pemphigus vulgaris, pemphigus foliaceous, epidermolysis bullosa acquisita, linear IgA disease etc.

3. Bullous SLE (BSLE), characterized by distinctive combination of clinical, histologic and immunopathologic features. ${ }^{5}$

Pedro and Dahl described the first case of bullous SLE in 1973. The differentiation between primary autoimmune blistering diseases in association with SLE and BSLE is open to question. ${ }^{6}$ The association of bullous SLE with lupus nephritis has been reported in adults. ${ }^{7} T 0$ the writer's knowledge, so far only a case of bullous SLE with seizure and nephritis is reported in a 13-year-old girl from India where renal biopsy was not done. ${ }^{8}$ We observed a young female who had bullous skin lesion and nephritis at first presentation of SLE and developed seizure during the same admission.

\section{CASE REPORT}

A 18 years girl from Lalitpur, Nepal was admitted to Tribhuvan University Teaching Hospital in early July 2012 with history of multiple painful, fluid filled tense lesions over bilateral axillae, oral ulcer and low grade fever of 4 days duration, decreased urine output amounting to oliguria and high coloured urine of one day duration and 3 episodes of epistaxis, scanty amount, one day prior to presentation. The skin lesions were pruritic and discrete and spared the anogenital regions initially. She also had arthralgia involving the large joints of limbs for 4 days. Seven days prior to presentation, she had taken ciprofloxacin for three days for sore throat and subsequently she was given amoxicillin and combination of ibuprofen and paracetamol for the skin lesions and fever which she continued till presentation. However, she did not have photosensitivity, hair loss, Raynaud's phenomenon, digital tip ulcers, chest pain, cough, hemoptysis, seizure or psychosis. She had no history of blistering lesions in the past, no history of similar lesions in her family.

On examination, she weighed $51 \mathrm{Kg}$, was pale, her pulse was 92/min, BP was $110 / 70 \mathrm{mmHg}$. She had multiple tense, clear fluid filled blisters ranging from 1 to $4 \mathrm{~cm}$ in bilateral axillae (figure1) and a few in the abdomen and lower limbs, not correlating with sun exposure. The bullae in axialle had erythematous base while those in the abdomen and legs had clear base. Nickolsky's sign was negative. She had oral ulcers mainly in the

\footnotetext{
* Corresponding author

Mahesh Raj Sigdel, $M D$

Department of Internal Medicine

TU Teaching Hospital, Kathmandu, Nepal

Email: maheshsigdel@hotmail.com
} 
inner aspect of lips, gums and tongue. Rest of the general and systemic examination was unremarkable.

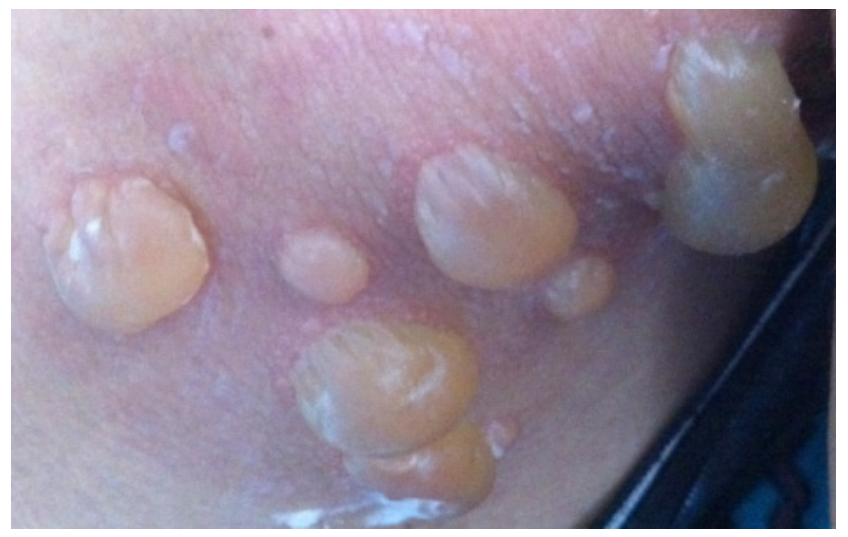

Figure 1: Multiple tense, clear fluid filled blisters

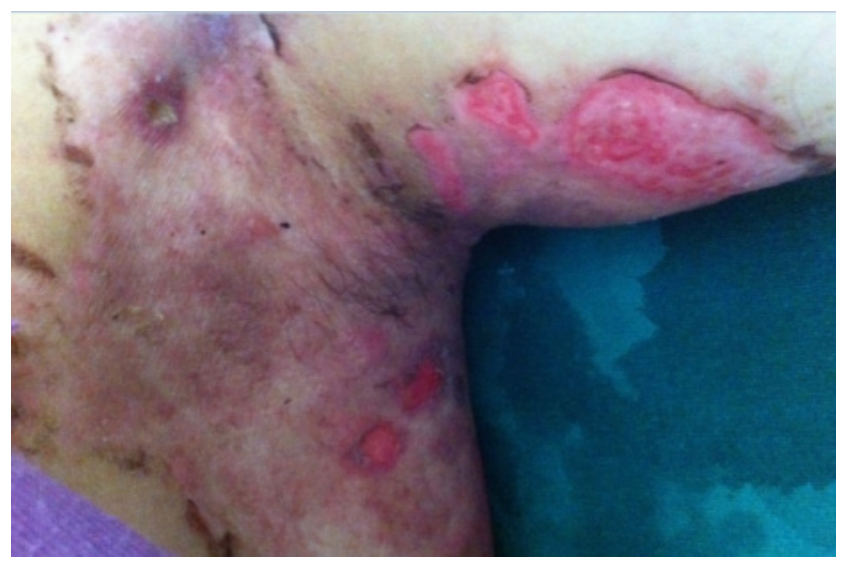

Figure 2: Wide erythematous denuded area caused by spontaneously ruptured bullae

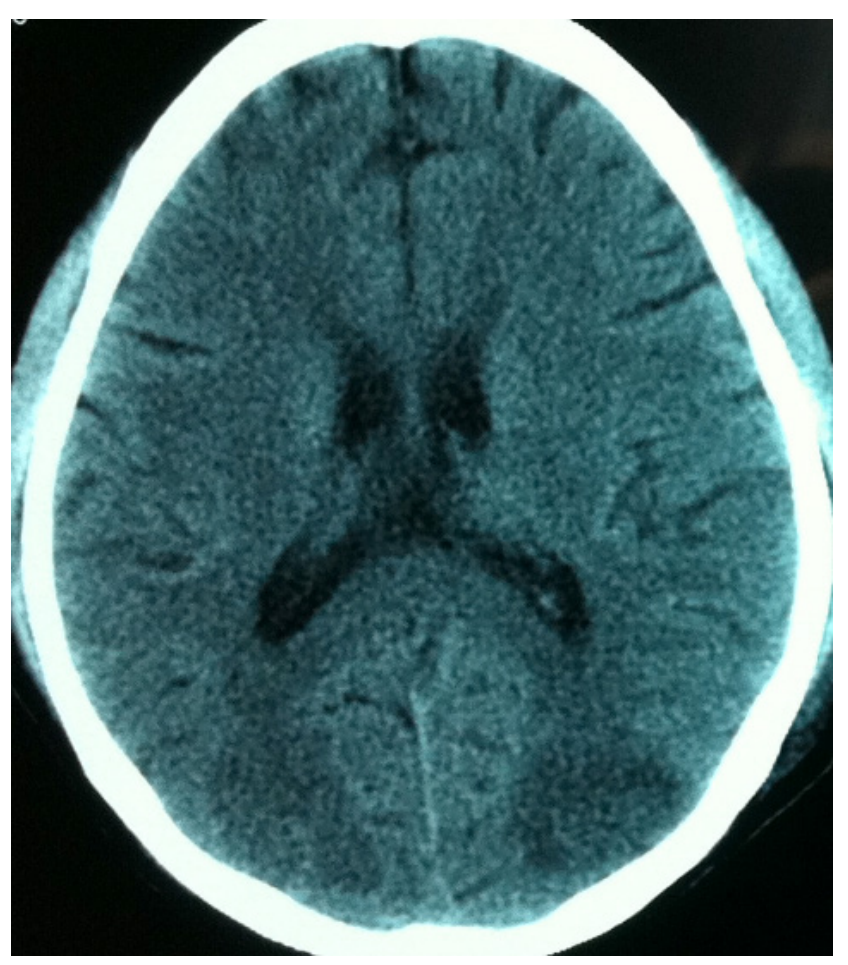

Figure 3: CT scan head, showing hypodense lesion in bilateral parietooccipital region, left>right
Initial investigations revealed $\mathrm{Hb}: 8.9 \mathrm{gm} / \mathrm{dl}$, total leucocyte count: 4900 /cumm, $\mathrm{N}_{68}, \mathrm{~L}_{29}$, platelet: 1,85,000/cumm, normal peripheral smear with absence of fragmented cells, urine R/M: albumin:,$++ \mathrm{RBC}$ : plenty/HPF, few of the red cells were dysmorphic, ultrasound abdomen: maintained kidney size, increased cortical echotexture and diminished corticomedullary differentiation, random blood sugar: $5 \mathrm{mmol} / \mathrm{L}$, blood urea: $8.3 \mathrm{mmol} / \mathrm{L}$, serum creatinine: $182 \mathrm{micro} \mathrm{mol} / \mathrm{L}$, serum albumin: $29 \mathrm{gm} / \mathrm{L}$; CPK, liver enzymes, septic profile \& coagulation profiles were normal. Her 24 hours urinary protein was 1.8 gm/day. Her HIV 1-2 antibody test, $\mathrm{HBsAg}$ and anti HCV tests were nonreactive by ELISA. ASO was negative $(<200 i \mathrm{u} / \mathrm{ml}), \mathrm{C}$ reactive protein (CRP) was,++ TORCH IgM negative, ANA (ANF) test: positive by ELISA, anti ds-DNA: $3 \mathrm{iu} / \mathrm{ml}$ by ELISA (negative), anti cardiolipin antibody IgG, IgM: negative, complement (C3): $33 \mathrm{mg} / \mathrm{dl}($ low), and chest X-ray was normal.

Her first skin biopsy report showed multiple intraepidermal bullae suggestive of differential diagnoses of drug induced pemphigus and pemphigus vulgaris. Repeat skin biopsy reported from another center showed intraepidermal blister with some acantholysis of spinous keratinocytes; the upper dermis was mildly edematous and had several neutrophils scattered close to the epidermis; IgG was strongly positive at dermoepidermal junction, IgA positive at dermoepidermal junction, IgM and C3 negative, and the diagnosis of IgA pemphigus was a possibility. Kidney biopsy revealed: focal necrotizing, diffuse proliferative glomerulonephritis with cellular crescents in $2 / 15$ (13.3\%) of sampled glomeruli. Direct immunofluorescence (DIF) revealed "full house" staining. Possibility of renal involvement in SLE was suggested.

This girl was managed in consultation with Dermatologist. She was initially treated with antibiotic and steroid, hydrocortisone, $50 \mathrm{mg}$ IV eight hourly which was subsequently increased to $100 \mathrm{mg}$ eight hourly and hydroxychloroquine was added after ANA was positive. The bullous lesion remitted partially but immediately new lesions appeared extending to palms, soles, anogenital region and ulcers in the oropharynx. Her serum creatinine came down to 149 micromol/L on fifth day of admission which subsequently rose to $273 \mathrm{micromol} / \mathrm{L}$ on $14^{\text {th }}$ day of admission. Once the skin biopsy report became available hydrocortisone was increased to $200 \mathrm{mg}$ IV eight hourly;inj cyclophosphamide $500 \mathrm{mg}$ IV, was given once renal biopsy report was available. Supportive care was continued. With these measures, further new crops of skin lesions did not appear and renal function improved gradually to the lowest of creatinine149 micromol/L on $25^{\text {th }}$ day of admission and just before discharge. The bullae spontaneously ruptured leaving behind wide erythematous denuded area (Fig 2). However, on the $21^{\text {st }}$ day of admission, she developed a generalized tonic clonic seizure with tongue bite. BP was 140/96 mmHg and metabolic parameters were normal at the time of seizure. CT scan head, revealed hypodense lesion in bilateral parieto-occipital region, left>right (Fig 3). However, regular anticonvulsants were not started after consultation with neurologist. On the $26^{\text {th }}$ day of admission, she was discharged walking home with support, skin lesions abated, kidney function improved and no further episodes of seizure. Her main medicines at discharge were prednisolone $70 \mathrm{mg} /$ day, hydroxychloroquine $400 \mathrm{mg}$ / day, cotrimoxazole prophylaxis, along with topical applications. At one week after discharge, she was improving, skin lesions resolving and creatinine came down to $117 \mathrm{micromol} / \mathrm{L}$.

\section{DISCUSSION}

Lupus has a multitude of skin manifestations. Early lesions of CLE are notoriously difficult to diagnose. It is old dermatology wisdom that lupus and syphilis are the great imitators. ${ }^{1}$ Lupus erythematosus (LE) associated skin lesions are classified as histopathologically lupus specific skin lesions 
(acute cutaneous LE, subacute cutaneous LEand chronic cutaneous LE) and histopathologically lupus non-specific skin lesions (egvasculitic lesions, alopecia, bullous lesions, urticaria, leg ulcers, erythema multiformeetc). ${ }^{1}$ Not all blisters in patients with lupus represent BSLE. ${ }^{4} \mathrm{~A}$ number of criteria have been suggested to diagnose BSLE. ${ }^{4,6,9}$ The essence of these include:

- A diagnosis of SLE based on ACR criteria. ${ }^{10}$

- An acquired, non-scarring bullous eruption arising on, but not limited to, sun-exposed skin.

- Asubepidermal blister with a predominantly neutrophilic infiltrate in the dermis and at the basement membrane zone.

- Direct immunofluorescence (DIF) of perilesional skin showinglgG, IgA, IgM and $\mathrm{C} 3$ deposits at dermoepidermal junction.

- Indirect immunoflourescence-negative or positive for circulating BMZ autoantibodies to type VII collagen

Our patient fulfilled ACR criteria for diagnosis of SLE (oral ulcers, ANA positivity, biopsy proven lupus nephritis and lupus cerebritis). Decreased complement level supported the diagnosis. The drugs she had taken prior to presentation are not usually incriminated in drug induced lupus. ${ }^{11}$ However, the biopsy of the skin revealed intraepidermal blister with IgG and IgA deposits without $\mathrm{C} 3$ deposits suggesting a differential diagnoses other than BSLE.These together lead us to diagnose SLE with autoimmune bullous disease, though anti-dsDNA was negative. Other tests like anti-histone antibody, anti-smith antibody, anti-Ro, anti-La etc were not done because of their local unavailability.

The odds for clinically manifest internal organ involvement in the setting of CLE are highly variable and are related to the type of CLE. Acute cutaneous LE (Lupus specific) has a strong association with systemic disease and non-specific skin lesions always indicate disease activity. ${ }^{12}$ Literature describesassociation of BSLE with lupus nephritis and bullous lesions predating disease flare in patient with previously diagnosed SLE; only a few report bullous lesions as the first presentation of SLE. ${ }^{7,13}$ To our knowledge, ours is the first case of biopsy proven lupus nephritis, seizure and bullous lesions at the first presentation of SLE, however the exact cause of blisters could not be ascertained. The difficulty in making exact diagnosis of bullous disease in SLE and the controversy regarding their nomenclature has been described in the literature. .,14,15 $^{-14}$

The goal in managing blistering disease is to decrease formation of blisters,

\section{REFERENCES}

1. Obermoser G, Sontheimer RD, Zelger B. Overview of common, rare and atypical manifestations of cutaneous lupus erythematosus and histopathological correlate. Lupus 2010;19:1050-70.

2. Albrecht J, Berlin JA, Braverman IM, et al. Dermatology position paper on the revision of the 1982 ACR criteria for systemic lupus erythematosus. Lupus 2004;13:839-49.

3. Grönhagen CM, Gunnarsson I, Svenungsson E, et al. Cutaneous manifestations and serological findings in 260 patients with systemic lupus erythematosus. Lupus 2010;19:1187.

4. Camisa C, Sharma HM. Vesiculobullous systemic lupus erythematosus. J Am Acad Dermatol 1983;9:924-33. promote healing, and prevent scarring.The majority of patients with pemphigus and pemphigoid respond to high-dose systemic corticosteroids and immunosuppressive agents. Patients who do not respond are treated with Dapsone, intravenous immunoglobulinor rituximab. In one study, the overall response rates to dapsone, either alone or in combination with corticosteroids or immunosuppressive agents, were $84 \%$ in mucous membrane pemphigoid, and $81 \%$ in bullous pemphigoid. ${ }^{16}$

Treatments for cutaneous LE include:17

1. Preventive (photoprotective) strategies: application of sunscreens containing titanium dioxide or zinc oxide (physical blocking agents) and avobenzone or ecamsule (chemical blocking agents) of sun protection factor $\geq 30$ protecting against both UVA and UVB.

2. Topical therapies: corticosteroids and calcineurin inhibitors

3. Systemic therapies: oral aminoquinoloneantimalarials (hydroxychloroquine, chloroquine, mepacrine) make the first line.$^{18}$ Additionalsystemic therapies may be conventional treatments (corticosteroids,methotrexate, thalidomide, retinoids, dapsone, azathioprine) and newer immunomodulatory therapies(efalizumab, anti-tumor necrosis factor agents, intravenous immunoglobulin, rituximab).

Dapsone is the initial treatment of choice for BSLE.The response is usually dramatic, with cessation of new blister formation within 1-2 days and rapid healing of existing lesions. . $^{17,19}$

The difficulty to pin point the diagnosis is reflected in the management of the present case as well. Diagnosis of SLE was firmly establishedlater only. And skin biopsy suggested drug induced pemphigus, pemphigus vulgaris or IgA pemphigus. Hence she was initially given low dose steroid with hydroxychloroquineand only later given high dose steroid and cyclophosphamide along with topical therapy to which both her skin lesions and renal function improved. She was planned for pulse monthly iv cyclophosphamide with steroid, ${ }^{20}$ and dapsone,${ }^{16,17}$ should her skin lesions relapse. However she was lost to follow up.

\section{CONCLUSION}

SLE should be suspected in any young female patient with bullous skin lesions. Though it may not be bullous SLE, other blistering autoimmune diseases could be associated. Dapsone is the drug of choice for bullous SLE.

5. Yell JA, Wojnarowska F. Bullous skin disease in lupus erythematosus. Lupus 1997;6:112-21.

6. Yell JA, Allen J, Wojnarowska F, et al. Bullous systemic lupus erythematosus: revised criteria for diagnosis. $\mathrm{Br} J$ Dermatol 1995;132:921-8.

7. Ng YY, Chang IT, Chen TW, et al. Concomitant lupus nephritis and bullous eruption in systemic lupus erythematosus. Nephrol Dial Transplant 1999;14:1739-43.

8. Kumar S, Agarwal I. Unusual presentation of childhood Systemic Lupus Erythematosus. Pediatric Rheumatology 2007;5:20.

9. Gammon WR, Briggaman RA. Bullous SLE: a phenotypically distinctive but immunologically heterogeneous bullous disorder. J Invest Dermatol 1993;100:S28-34. 
10. Hochberg MC. Updating the American College of Rheumatology revised criteria for the classification of systemic lupus erythematosus. Arthritis Rheum 1997;40:1725.

11. Marzano AV, Vezzoli P, Crosti C. Drug-induced lupus: an update on its dermatologic aspects. Lupus 2009;18:935.

12. Patel P, Werth V. Cutaneous lupus erythematosus: a review. Dermatol Clin 2002;20:373-85.

13. Fujimoto W, Hamada T, Yamada J, et al.Bullous Systemic Lupus Erythematosus as an Initial Manifestation of SLE. Dermatol 2005;32:1021-7.

14. Sansaricq F, Stein SL, Petronic-Rosic V. Autoimmune bullous diseases in childhood. Clin Dermatol 2012;30:114-27.

15. Ljubojevic S, Lipozenčić J. Autoimmune bullous diseases associations. Clin Dermatol 2012;30:17-33.
16. Gürcan HM, Ahmed AR. Efficacy of Dapsone in the Treatment of Pemphigus and Pemphigoid Analysis of Current Data. Am J Clin Dermatol 2009;10:383-96.

17. Walling HW, Sontheimer RD. Cutaneous Lupus Erythematosus Issues in Diagnosis and Treatment. Am J Clin Dermatol 2009;10:365-81.

18. WozniackaA, McCauliffe DP. Optimal Use of Antimalarials in Treating Cutaneous Lupus Erythematosus. Am J Clin Dermatol 2005;6:1-11.

19. Kuhn A, Ochsendorf F, Bonsmann G. Treatment of cutaneous lupus erythematosus. Lupus 2010;19:1125-36.

20. Ponticelli C, Glassock RJ, Moroni G. Induction and maintenance therapy in proliferative lupus nephritis. J Neprol 2010;23:9-16. 